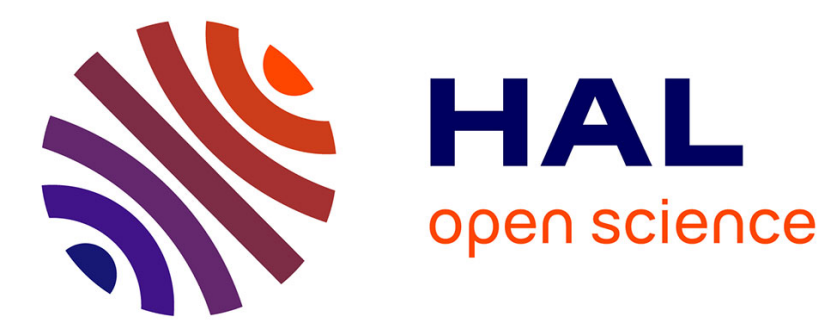

\title{
Mathematical and numerical study of a dusty knudsen gas mixture: extension to non-spherical dust particles Frédérique Charles
}

\section{To cite this version:}

Frédérique Charles. Mathematical and numerical study of a dusty knudsen gas mixture: extension to non-spherical dust particles. Springer INdAM Series, 2021, Springer INdAM Series, 48, pp.129-145. 10.1007/978-3-030-82946-9_6. hal-03002236v2

\section{HAL Id: hal-03002236 \\ https://hal.science/hal-03002236v2}

Submitted on 18 Nov 2020

HAL is a multi-disciplinary open access archive for the deposit and dissemination of scientific research documents, whether they are published or not. The documents may come from teaching and research institutions in France or abroad, or from public or private research centers.
L'archive ouverte pluridisciplinaire HAL, est destinée au dépôt et à la diffusion de documents scientifiques de niveau recherche, publiés ou non, émanant des établissements d'enseignement et de recherche français ou étrangers, des laboratoires publics ou privés. 


\title{
Mathematical and numerical study of a dusty knudsen gas mixture : extension to non-spherical dust particles
}

\author{
Frédérique Charles
}

\begin{abstract}
In this work, we consider the model introduced in 7] describing the movement of dust particles in a very rarefied atmosphere. The gas is treated as a Knudsen gas, whereas the interaction between dust particles and gas molecules is modeled by considering a moving domain free transport equation (including the boundary with the particles and the boundary of the domain). We here precise the proof of existence of solutions to the initial-boundary value problem annonced in [7. Moreover, we introduce a new numerical strategy, based on a splitting between the transport of the gas molecules and the movement of the boundary. This strategy allow to perform 2d-numerical simulations with elliptical-shaped particles.
\end{abstract}

\section{Introduction}

We considere here a mixture of a rarefied gas and macroscopic particles (such as dust particles). A typical example of such a situation is the study of the dynamics of gases inside a microelectromechanical system (MEMS). More precisely, we place ourselves in the physical situation described by the order of magnitude of the physical constant in Table 1 Under these assumptions, the mean free path

\begin{tabular}{|ccc|}
\hline Temperature of the gas & $\mathrm{T}_{g}$ & $293 \mathrm{~K}$ \\
Mach number & $\mathrm{Ma}$ & 0.1 \\
Gas Pressure & $\mathrm{P}$ & $5 \mathrm{~Pa}$ \\
Size of the Container & $\mathrm{L}$ & $2 \cdot 10^{-4} \mathrm{~m}$ \\
Radius of particles & $\mathrm{r}$ & $10^{-5} \mathrm{~m}$ \\
\hline
\end{tabular}

Table 1: Order of magnitude of physical quantities in the situation under study

of the gas is equal to $\lambda_{g}=2 \cdot 10^{-3} \mathrm{~m}$, and the Knudsen number of the gas (that is, the ratio between the mean free path and the charateristic length of the domain) inside the container is $K_{n}=10$. In this context, a kinetic description of the gas is more suitable that a description with fluid models. Moreover, one of the advantages of kinetic models is that they depend much less on phenomenologic laws than most models of continuum mechanics. We therefore consider a mesoscopic scale and describe the gas thanks to a density function defined in the phase space (no distinction is made here between the different types of 
molecules constituting the gas). Without any particules, a rarefied gas inside a vessel could typically be described by the Boltzman equation (see [4]) with suitable boundary conditions. A kinetic description of a gas-particle mixture was introduced in [5], where the flow of particles is described thanks to another density function, and interactions between particules and molecules are modeled by integral collisions operators. We can also mention [11, where the movement of spherical particles is described through equations on their momentum and velocity, and where the gas is described by a Boltzmann equation with an integral operator describing gas-particles interactions. In [13, the motion of a rigid body immersed in a gas is governed by the Newton-Euler equations, where the force and the torque on this body are computed from the momentum transfer of the gas molecules colliding with the body ; the gas is described by a Boltzmann equation without any effect of the body on the gas.

The point of view adopted in [7] is rather different. The interaction between the gas and the particles (in finite number) is modeled by considering the evolution of the gas in a moving domain, where the boundary of the domain include the surface of the particules. This approach has already been introduced in [9] and $[12$. However, in the later works, authors use an eulerian numerical method (Finite-Difference and Semi-Lagrangien method respectively) which makes the treatment of boundary conditions rather complicated; the numerical study is therefore only carried out in dimension 1.

Moreover, for large Knudsen number (typically larger to 10), it is generally admitted [10] that the gas can be considered as a Knudsen gas (or molecular flow), and we therefore neglect here collisions between molecules. Theorical studies of the convergence to equilibrium of a particle in a Knudsen gas have been carried out in [2] and [3], but no numerical simulation has been performed. The study of a Knudsen system in a moving domain, both at the theoretical and at the numerical level, has been the subject of [8, but in the context of a gas in a vessel with absorbing boundary conditions.

The paper is organised as follows. We first recall the model introduced in [7] for spherical particles, that we extend to any shape of particle. We then precise the proof of the existence of solutions announced in [7]. Finally we present a new numerical strategy, which allow to perform numerical simulations with non-spherical particles, and some scenarios of numerical simulations.

\section{Description of the model}

We briefly recall the model introduced in [7] and generalize it to non-spherical particles. We consider a free transport equation in a open bounded spatial domain $D \subset \mathbb{R}^{d}, d \in \mathbb{N}^{*}$, which describe the evolution of the molecules density $f:=f(t, x, v)$, with $(t, x, v) \in \mathbb{R}^{+} \times D \times \mathbb{R}^{d}$. The motion of particles is supposed to be known, and we denote $B_{i}(t)$ the closed set corresponding to the region occupied by the particle indexed by $i$ at time $t$. We introduce the time $\mathcal{T}_{1}$ which guarantee the non-overlapping of particles

$$
\mathcal{T}_{1}=\sup \left\{t \geq 0: \forall s \in\left[0, t\left[, B_{j}(s) \cap B_{i}(s)=\emptyset \quad \text { for all } j, i=1, \ldots, N_{d}, j \neq i\right\}\right.\right.
$$

and the time $\mathcal{T}_{2}$ which guarantee the non-exit of particles out of the domain

$$
\mathcal{T}_{2}=\sup \left\{t \geq 0: \forall s \in\left[0, t\left[, B_{i}(s) \cap \partial D=\emptyset \quad \text { for all } i=1, \ldots, N_{d}\right\} .\right.\right.
$$




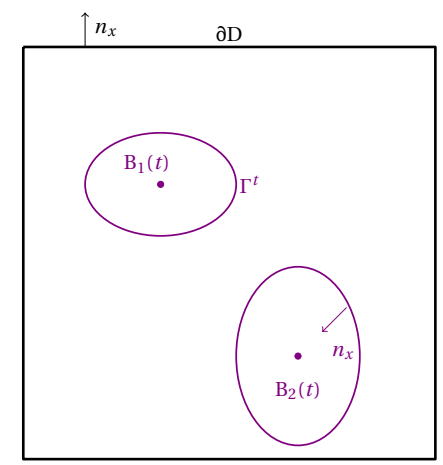

Figure 1: Graphical description of the problem

We do not consider here collisions of a particle with another particle or with the boundary of the domain, and therefore consider the problem for $t \in[0, \mathcal{T})$, with $\mathcal{T} \leq \min \left(\mathcal{T}_{1}, \mathcal{T}_{2}\right)$. For $t \in[0, \mathcal{T})$ we denote $\Omega^{t}$ the domain occuped by the gas at time $t$

$$
\Omega^{t}:=D \backslash \bigcup_{i=1}^{N_{d}} B_{i}(t),
$$

and $\partial \Omega^{t}=\partial D \cup \Gamma^{t}$ its boundary, with

$$
\Gamma^{t}=\bigcup_{i=1}^{N_{d}} \partial B_{i}(t)
$$

(see Figure 1). The motion of the domain is described through the velocity law of each point of the boundary at a given time $t$. We define a field $c$ : $\mathbb{R}^{+} \times \mathbb{R}^{d} \rightarrow \mathbb{R}^{d}$ which gives the local velocity of each point $x \in \partial \Omega^{t}$, for any $t \in[0, \mathcal{T}[$. We note that for any $x \in \partial D$, we have $c(t, x)=0$. We assume that the interaction between molecules and particles is described by a diffuse reflection on the surface of the particle, and that all particles have the same temperature $T_{p}>0$, uniform on the surface. Following this assumption, the boundary condition on the surface of particles, that is for $x \in \Gamma^{t}$, writes

$$
f(t, x, v)=\left\{\begin{array}{c}
\int_{\left\{(w-c(t, x)) \cdot n_{x} \geq 0\right\}} k_{d, T_{p}}(x, v-c(t, x), w-c(t, x)) f(t, x, w) d w \\
\quad \text { for } x \in \Gamma^{t},(v-c(t, x)) \cdot n_{x}<0 \\
0 \quad \text { for } x \in \Gamma^{t},(v-c(t, x)) \cdot n_{x} \geq 0
\end{array}\right.
$$

where $n_{x} \in \mathbb{S}^{d-1}$ the outward normal originated in $x$, and $k_{d, T_{p}}$ a kernel modelling a diffuse reflexion at temperature $T_{p}$, defined by (see [14])

$$
k_{d, T_{p}}(x, v, w)=\sqrt{\frac{2 \pi}{T_{p}}} \mathcal{M}_{T_{p}}(v)\left(w \cdot n_{x}\right),
$$

where $\mathcal{M}_{T_{p}}$ is the centered Maxwellienne at temperature $T_{p}$ :

$$
\mathcal{M}_{T_{p}}(v)=\frac{1}{\left(2 \pi T_{p}\right)^{d / 2}} e^{-\frac{|v|^{2}}{2 T_{p}}} .
$$


This kernel verifies the following property

$$
\int_{\left\{w \cdot n_{x} \geq 0\right\}} k_{d, T_{p}}(x, v, w) M_{T_{p}}(w) d w=M_{T_{p}}(v) .
$$

For $x \in \partial D$, that is on the boundary of the external domain, which is assumed to be still $(c(t, x)=0$ for $x \in \partial D)$, the boundary condition writes

$$
f(t, x, v)= \begin{cases}\int_{\left\{w \cdot n_{x} \geq 0\right\}} k(x, v, w) f(t, x, w) d w & \text { for } x \in \partial D, v \cdot n_{x}<0 \\ 0 & \text { for } x \in \partial D, v \cdot n_{x} \geq 0\end{cases}
$$

where $k$ is a kernel modeling an accommodation reflexion on $\partial D$ at temperature $T_{p}$

$$
k(x, v, w)=\gamma k_{s}(x, v, w)+(1-\gamma) k_{d, T_{p}}(x, v, w)
$$

where $\gamma \in[0,1]$ is the accommodation coefficient, and $k_{s}$ a kernel modeling a specular reflexion

$$
k_{s}(x, v, w)=\delta\left(w-v+2\left(v \cdot n_{x}\right) n_{x}\right) .
$$

The kernel $k_{s}$ verifies, for all function $\varphi$ defined on $\mathbb{R}^{+}$:

$$
\int_{\left\{w \cdot n_{x} \geq 0\right\}} k_{s}(x, v, w) \varphi(|w|) d w=\varphi(|v|) \mathbb{1}_{\left\{v \cdot n_{x} \leq 0\right\}} .
$$

One can summarize the boundary conditions by

$$
f(t, x, v)=\int_{\mathbb{R}^{3}} K(t, x, v, w) f(t, x, w) d w \mathbb{1}_{\left\{(v-c(t, x)) \cdot n_{x}<0\right\}} \quad \text { for } x \in \partial \Omega^{t},
$$

with

$$
K(t, x, v, w)= \begin{cases}k_{d, T_{p}}(x, v-c(t, x), w-c(t, x)) \mathbb{1}_{\left\{w-c(t, x) \cdot n_{x} \geq 0\right\}} & \text { if } x \in \Gamma^{t} \\ \left(\gamma k_{s}(x, v, w)+(1-\gamma) k_{d, T_{p}}(x, v, w)\right) \mathbb{1}_{\left\{w \cdot n_{x} \geq 0\right\}} & \text { if } x \in \partial D .\end{cases}
$$

We end-up with the following model

$$
\frac{\partial f}{\partial t}+v \cdot \nabla_{x} f=0 \quad(t, x, v) \in \mathbb{R}^{+} \times \Omega^{t} \times \mathbb{R}^{d},
$$

with the initial condition

$$
f(0, x, v)=f^{\text {in }}(x, v) \mathbb{1}_{\left\{\Omega^{0} \times \mathbb{R}^{d}\right\}}(x, v)
$$

and the boundary conditions $(8)-(9)$.

\section{$3 \quad$ Existence of solutions}

We slightly modify and precise the Theorem 3.3 in [7].

Theorem 3.1. Let $\mathcal{T} \in\left(0, \min \left(T_{1}, T 2\right)\right)$, where $T_{1}$ and $T_{2}$ are defined by (1) and (2). We assume that $c \in \mathrm{E}^{\infty}((0, T) \times D)$. Let $f^{\text {in }} \in L^{\infty}\left(\Omega^{0} \times \mathbb{R}^{d}, e^{|v|^{2} / T_{p}} \mathrm{~d} v \mathrm{~d} x\right)$ $f^{\text {in }} \geq 0$ for a.e. $(x, v) \in \Omega^{0} \times \mathbb{R}^{d}$. Then there exists at least one non-negative weak solution $f \in L^{\infty}\left((0, T) ; L^{\infty}\left(\bar{\Omega}^{t}, \mathbb{R}^{d}\right)\right)$ of the initial-boundary value problem (10) - (11) - (8) - Moreover $(t, x, v) \mapsto f(t, x, v) e^{\frac{|v-c(t, x)|^{2}}{2 T_{p}}} \in L^{\infty}\left((0, T) ; L^{\infty}\left(\bar{\Omega}^{t}, \mathbb{R}^{d}\right)\right)$. 
Proof. We follow and adapt the proof for a fixed domain made in [1]. We first consider the auxiliary problem for the function $g: \mathbb{R}^{+} \times \Omega^{t} \times \mathbb{R}^{d} \rightarrow \mathbb{R}$

$$
\frac{\partial g}{\partial t}+v \cdot \nabla_{x} g=0, \quad(t, x, v) \in \mathbb{R}^{+} \times \Omega^{t} \times \mathbb{R}^{d},
$$

with initial data

$$
g(0, x, v)=f^{\text {in }}(x, v) \mathbb{1}_{\left\{\Omega^{0} \times \mathbb{R}^{d}\right\}}(x, v)
$$

and boundary conditions

$$
g(t, x, v)=\Phi(t, x, v) \mathbb{1}_{\left\{(v-c(t, x)) \cdot n_{x}<0\right\}}
$$

for a.e. $(x, v) \in \partial \Omega^{t} \times \mathbb{R}^{d}$, where $\Phi \in L^{\infty}\left((0, T) \times \partial \Omega^{t} \times \mathbb{R}^{d}\right)$ is a given function. The problem (12)- $(13)$ - (14) has a unique weak solution, given by the method of characteristics

$$
g(t, x, v)=f^{\mathrm{in}}(x-v t, v) \mathbb{1}_{\left\{\tau_{\Omega^{t}}(x, v)>t\right\}}+\Phi\left(t, x-\tau_{\Omega^{t}}(x, v) v, v\right) \mathbb{1}_{\left\{\tau_{\Omega^{t}}(x, v)<t\right\}},
$$

where

$$
\tau_{\Omega^{t}}(x, v)=\left\{\begin{array}{l}
+\infty \quad \text { if }\left\{\theta>0: x-\theta v \in \Gamma^{t-\theta} \cup \partial D\right\}=\emptyset \\
\inf \left\{\theta>0: x-\theta v \in \Gamma^{t-\theta} \cup \partial D\right\} \quad \text { otherwise. }
\end{array}\right.
$$

$\tau_{\Omega^{t}}(x, v)$ correspond to the arrival time on the boundary when we follow backward the charateristic starting from $x \in \Omega^{t}$ at velocity $v \in \mathbb{R}^{d}$. We deduce that

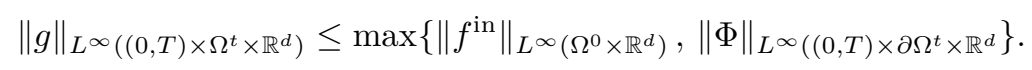

We now consider the sequence $\left(f_{n}\right)_{n \in \mathbb{N}}$ of functions, such that

$$
f_{0}(t, x, v)=0 \quad \text { for a.e. }(t, x, v) \in[0, T) \times \bar{\Omega}^{t} \times \mathbb{R}^{d}
$$

and, for all $n \in \mathbb{N}, n \geq 1, f_{n}$ is the solution of the following initial-boundary value problems:

$$
\frac{\partial f_{n}}{\partial t}+v \cdot \nabla_{x} f_{n}=0, \quad(t, x, v) \in \mathbb{R}^{+} \times \Omega^{t} \times \mathbb{R}^{d},
$$

with initial data

$$
f_{n}(0, x, v)=f^{\mathrm{in}}(x, v) \mathbb{1}_{\left\{\Omega^{0} \times \mathbb{R}^{d}\right\}}(x, v)
$$

and boundary conditions

$$
f_{n}(t, x, v)=\int_{\mathbb{R}^{3}} K(t, x, v, w) f_{n-1}(t, x, w) d w \mathbb{1}_{\left\{(v-c(t, x)) \cdot n_{x}<0\right\}}
$$

for $(x, v) \in \partial \Omega^{t} \times \mathbb{R}^{d}$, where $K$ is defined in (9). Thanks to properties (5) and (7), the boundary condition (18) lead to the estimate on the boundary

$$
\left\|\frac{f_{n}}{\mathcal{M}_{T_{p}}(v-c(t, x))}\right\|_{L^{\infty}\left((0, T) \times \partial \Omega^{t} \times \mathbb{R}^{d}\right)} \leq\left\|\frac{f_{n-1}}{\mathcal{M}_{T_{p}}(v-c(t, x))}\right\|_{L^{\infty}\left((0, T) \times \partial \Omega^{t} \times \mathbb{R}^{d}\right)}
$$


Then the estimate (15) allow to prove by induction that

$$
\left\|\frac{f_{n}}{\mathcal{M}_{T_{p}}(v-c(t, x))}\right\|_{L^{\infty}\left((0, T) \times \bar{\Omega}^{t} \times \mathbb{R}^{d}\right)} \leq\left\|\frac{f^{\text {in }}}{\mathcal{M}_{T_{p}}(v-c(t, x))}\right\|_{L^{\infty}\left((0, T) \times \Omega^{0} \times \mathbb{R}^{d}\right)}
$$

Moreover, an immediate induction argument prove that $f_{n} \geq 0$ for all $n \geq 0$. We obtain

$$
0 \leq f_{n}(t, x, v) \leq f_{n}(t, x, v) e^{\frac{|v-c(t, x)|^{2}}{2 T_{p}}} \leq\left\|f^{\text {in }} e^{\frac{|v|^{2}}{T_{p}}}\right\|_{L^{\infty}\left(\Omega^{0} \times \mathbb{R}^{d}\right)} e^{\frac{\|c\|_{\infty}^{2}}{T_{p}}}
$$

for a.e. $(t, x, v) \in[0, T) \times \bar{\Omega}^{t} \times \mathbb{R}^{d}$. We then can proove that the sequence is non decreasing. Consider the sequence $h_{n}:=f_{n+1}-f_{n}$, for all $n \geq 0$. By linearity, for all $n \geq 0, h_{n}$ satisfy the free transport equation 12 with initial condition

$$
\forall(x, v) \in \Omega^{0} \times \mathbb{R}^{d}, \quad\left\{\begin{array}{l}
h_{0}(0, x, v)=f^{\text {in }}(x, v) \mathbb{1}_{\left\{\Omega^{0} \times \mathbb{R}^{d}\right\}}(x, v) \\
h_{n}(0, x, v)=0 \text { for } n \geq 1
\end{array}\right.
$$

and boundary condition

$$
h_{n}(t, x, v)=\int_{\mathbb{R}^{3}} K(t, x, v, w) h_{n-1}(t, x, w) d w \mathbb{1}_{\left\{(v-c(t, x)) \cdot n_{x}<0\right\}}
$$

for $(x, v) \in \partial \Omega^{t} \times \mathbb{R}^{d}$. We deduce that $h_{n}(t, x, v) \geq 0$ for a.e. $(t, x, v) \in$ $[0, T) \times \bar{\Omega}^{t} \times \mathbb{R}^{d}$. We have hence built a monotone non-decreasing sequence $\left(f_{n}\right)_{n \in \mathbb{N}}$ composed by non-negative and uniformly bounded functions a.e. in the domain of definition of the problem. By consequence, the sequence $\left(f_{n}\right)_{n \in \mathbb{N}}$ pointwise converges to a limit $f$, which is by construction a non-negative solution of the initial-boundary value problem $(\sqrt{10})-(11)-(8)$, and we can pass to the limit in estimate 21].

\section{Numerical simulations}

\subsection{Numerical method}

We describe here a new strategy for the numerical study of the model $(10)-(11)-$ (8), which is a modification of the particle method proposed in [7. The initial density $f^{\text {in }}$ of the gas is discretized by mean of a collection of weighted smooth shape functions centered on the particle positions, that is

$$
f_{\varepsilon, N_{m}}^{\text {in }}(x, v)=\sum_{k=1}^{N_{m}} \omega_{k} \varphi_{\varepsilon}\left(x-x_{k}^{0}\right) \varphi_{\varepsilon}\left(v-v_{k}^{0}\right),
$$

where $N_{m}$ represents the number of numerical particles, $\omega_{k}$ is the weight of the $k$-th numerical particle (which represent $\omega_{k}$ molecules). In (23), the shape function $\varphi_{\varepsilon}(x)=\varphi\left(\varepsilon^{-1} x\right) / \varepsilon^{d}$ is a smooth function with compact support. The term "numerical particles" is here used for avoiding any confusion with the (real) number of dust particles. Once the number $N_{m}$ of numerical particles has been chosen, the initial positions $\left(x_{k}^{0}\right)_{1 \leq k \leq N_{m}}$ and velocities $\left(v_{k}^{0}\right)_{1 \leq k \leq N_{m}}$ are sampled according to the initial density $f^{\mathrm{in}}$ (either in a deterministic way, either thanks 
to a Monte-Carlo procedure). Then, the positions and velocities of the numerical particles evolve in time by taking into account the different phenomena listed below:

(i) the free flow of the numerical particles in the absence of any interaction, mathematically represented by the transport operator $v \cdot \nabla$;

(ii) the boundary condition on $\partial D$; we can considere here specular reflexion or accommodation reflexion, but also a periodic condition in order to hide the effects of the boundary.

(iii) the diffuse reflexion between gas molecules and dust particles;

(iv) the time evolution of the set of dust particles.

We introduce a time discretization of step $\Delta t$ and we set $t^{n}=n \Delta t$. The density of gaseous molecules at time $t^{n}$-i.e. $f\left(t^{n}, \cdot, \cdot\right)$ where $f$ is the solution of $(10)$ (11)-(8) is then approached by

$$
f_{\varepsilon, N_{m}}^{n}(x, v)=\sum_{k=1}^{N_{m}} \omega_{k} \varphi_{\varepsilon}\left(x-x_{k}^{n}\right) \varphi_{\varepsilon}\left(v-v_{k}^{n}\right),
$$

where $\left(x_{k}^{n}\right)_{1 \leq k \leq N}$ and $\left(v_{k}^{n}\right)_{1 \leq k \leq N}$ are the positions and the velocities of the numerical particles at time $t^{n}$.

In [7, our strategy was to compute simultaneously the steps (i), (iii), (iv) previously described. For that purpose, we compute for each numerical particle the position of the possible intersection of its trajectory with the dust particle during the time $\Delta t$. To do that, we computed if the condition

$$
\min _{1 \leq i \leq N_{p}} \min _{t \in\left[t^{n}, t^{n}+\Delta t\right]}\left\|\xi_{i}(t)-x_{k}^{n}(t)\right\| \leq r
$$

is verified or not, where $\xi_{i}(t)$ is the position of the center of the spherical particle, $r$ its radius, and $x_{k}^{n}(t)=x_{k}^{n}+\left(t-t^{n}\right) v_{k}^{n}$ the trajectory of the numerical particle between time $t^{n}$ and $t^{n+1}$. However, this strategy is hardy adaptable to nonspherical particles.

We consider here a splitting between the advection stage of the dust particles (iv) and the evolution of gas molecules, corresponding to stages (i)-(ii)-(iii). In other word, we first transport dust particles independently of molecules during the time $\Delta t$, and we then transport numerical particles and perform the treatment of the boundary conditions. We thus come back to dealing with conditions at the boundaries of a fixed domain instead of a mobile domain. We first test on every numerical particle if $x^{n+1} \in \Omega^{t}$, and otherwise we compute the boundary condition. To do so, we only need a cartesian equation of the surface of dust particles, in order to calculate the intersection of this surface with a straight line as well as the normal vector at each point of the surface.

The latter strategy, which gives graphically similar results to the first one for spherical particles, allows to consider easily some ellipse-shaped particles. For such particles, our objective is in particular to observe the effect of the rotational velocity of the particle on the gas. This effect was not visible for spherical particles because the gas has no viscosity. 


\subsection{Numerical results}

We describe here a series of numerical experiments in dimension $d=2$. We suppose that the initial density is uniform in space and that it is described by a Maxwellian function in velocity, that is

$$
f^{\text {in }}(x, v)=f^{\text {in }}(v)=\frac{n_{0} m}{2 \pi k_{B} T^{\text {in }}} e^{-\frac{m\left|v-u_{g}\right|^{2}}{2 k_{B} T^{\text {in }}}}
$$

where $m$ is the mass of a gas molecule, $u^{\text {in }}$ and $T^{\text {in }}$ are respectively the initial macroscopic velocity and the temperature of the gas (in $\mathrm{K}$ ), and $n_{0}$ correspond to $\left\|f^{\text {in }}\right\|_{L^{1}\left(\Omega^{0} \times R^{3}\right)} /\left|\Omega^{0}\right|$. In this case, each component $\left(v_{k}^{0}\right)_{i}$, for $1 \leq k \leq N_{p}$ of the initial velocities of the gas particles is sampled according a gaussian law of mean $\left(u^{\text {in }}\right)_{i}$ and variance $k_{B} T^{\text {in }} / m$ (see [6] for details). The weights of the particles are identical, and are tuned in order to reproduce the mass of the initial condition:

$$
\omega_{k}=\frac{\left\|f^{\text {in }}\right\|_{L^{1}\left(\Omega^{0} \times R^{3}\right)}}{N_{p}}=\frac{n_{0}\left|\Omega^{0}\right|}{N_{p}}, \quad \text { for all } 1 \leq k \leq N_{p} .
$$

The initial positions of the numerical particles have been fixed on a regular grid, except inside the dust particles. In some scenarios, the gas has a macroscopic velocity along the first axis equal $u^{\text {in }}=V_{s} M_{a}$, where $M_{a}$ is the Mach number and $V_{s}$ is the sound velocity in air at temperature $T^{\text {in }}$. We take here $M_{a}=0.1$ and $T^{\text {in }}=293 \mathrm{~K}$; then $u^{\text {in }}=34.41 \mathrm{~m} / \mathrm{s}$. The temperature of the surface of particles is $500 \mathrm{~K}$. The value $n_{0}$ has been normalized to 1 . Indeed, the values of $n_{0}$ have no impact, neither on the transport of molecules and of dust particles (since these ones are no influenced by the surrounding gas) nor on the collisions between molecules and dust particles (the number of collisions is not computed as in DSMC methods). The domain $D$ is the square $[-1,1] \times[-1,1]$ (in $10^{-5}$ $\mathrm{m})$, with specular reflexion at the top and bottom boundary. We use a periodic boundary condition at the left and at the right sides of $\partial D$, in order to mimic an infinite domain in the $x$ direction. We use $B_{3}$-splines (see [6]) as shape functions $\varphi$, with a shape size $\varepsilon=h^{0.5}$, where $h$ is the initial distance between two numerical gas particles in each direction (and which is obviously linked to $\left.N_{m}\right)$.

Scenarios 1 and 2 The first simulations presents the rotation of a particle with no translational velocity. The particle is an ellipse, with axes equal to $a=2.5 \cdot 10^{-5}$ and $b=1 \cdot 10^{-5}$. In the first scenario, the macroscopic velocity of the gas is $u_{g}=(0,0)$, whereas in the second one the macroscopic velocity of the gas is $u_{g}=\left(-u^{\mathrm{in}}, 0\right)$. The rotational velocity of the particle is equal to $\Omega=2 \pi \cdot 10^{6} \mathrm{rad} / \mathrm{s}$ in both scenarios. Figures 2 and 3 show the time evolution of the number density $\rho(t, x)=\int_{\mathbb{R}^{3}} f(t, x, v) \mathrm{d} v$ of scenario 1 and 2 respectively. In particular, we can observe the effect of the macroscopic velocity of the gas, which acts as a side wind. Figure 4 show the comparison at a given time between the kinetic temperature of the gas

$$
T(t, x)=\frac{m}{2 k_{B} \rho(t, x)}\left(\int_{\mathbb{R}^{3}} f(t, x, v) v^{2} \mathrm{~d} v-\left|\frac{1}{\rho(t, x)} \int_{\mathbb{R}^{3}} f(t, x, v) v \mathrm{~d} v\right|^{2}\right)
$$


in scenarios 1 and 2. Here the macroscopic speed of the gas (which is much smaller than the kinetic velocity of molecules) does not have much influence on the temperature.
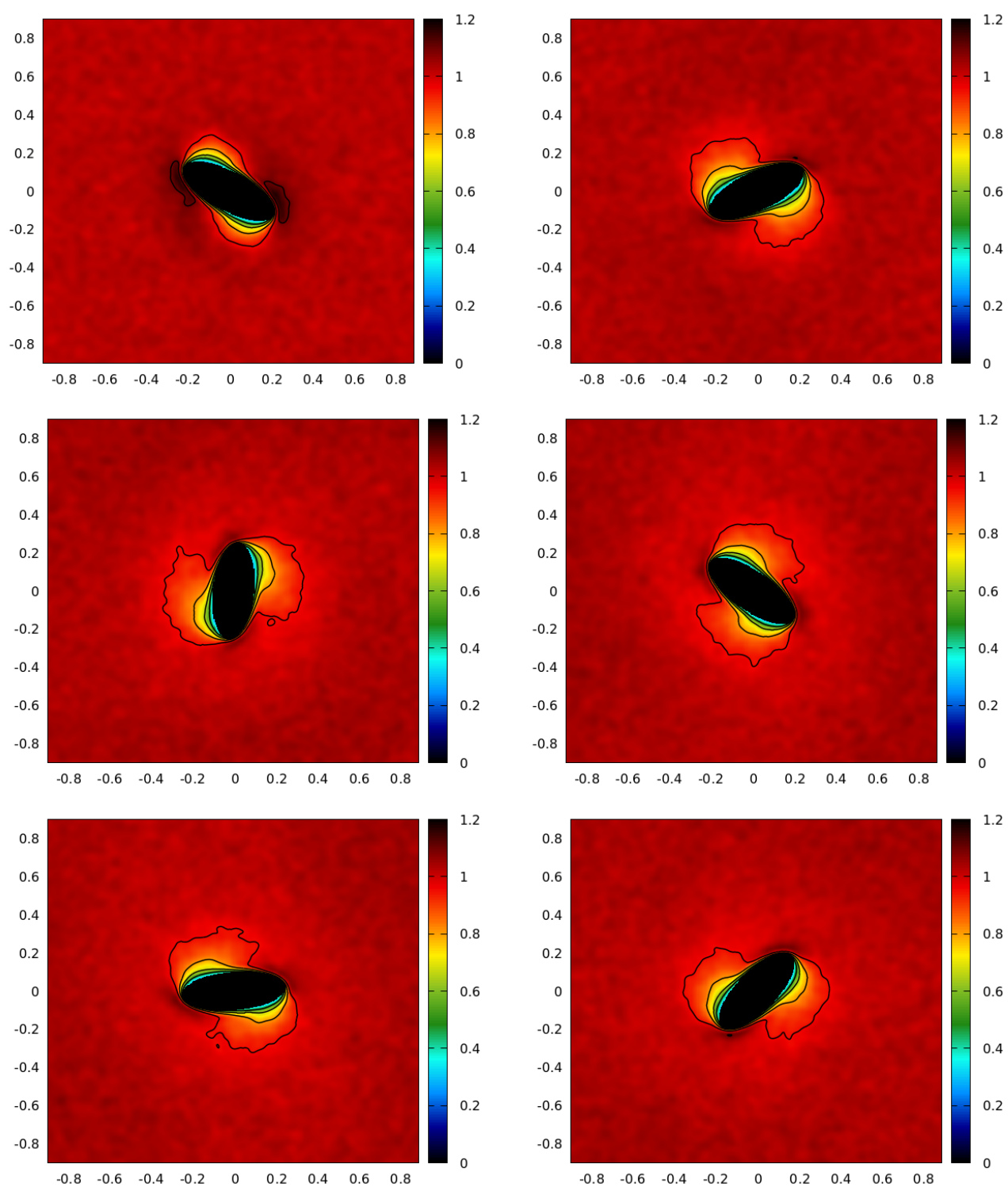

Figure 2: Time history of the gas density in Scenario 1, from left to right and from top to bottom, at times $8 \cdot 10^{-8} \mathrm{~s}, 2.4 \cdot 10^{-7} \mathrm{~s}, 4 \cdot 10^{-7} \mathrm{~s}, 5.6 \cdot 10^{-7} \mathrm{~s}, 7.2 \cdot 10^{-7}$ $\mathrm{s}, 8 \cdot 10^{-7} \mathrm{~s}$. The axis are scaled according to the length scale $L^{\circ}=10^{-4} \mathrm{~m}$.

Scenarios 3 and 4 In scenarios 3 and 4 , two particles are crossing each other with opposite velocities : $u_{p}^{1}=\left(0,2 u^{\text {in }}\right)$, and $u_{p}^{2}=\left(0,-2 u^{\text {in }}\right)$. The gas has a macroscopic velocity equal to $\left(-u^{\text {in }}, 0\right)$. In scenario 3 the dust particles have no 

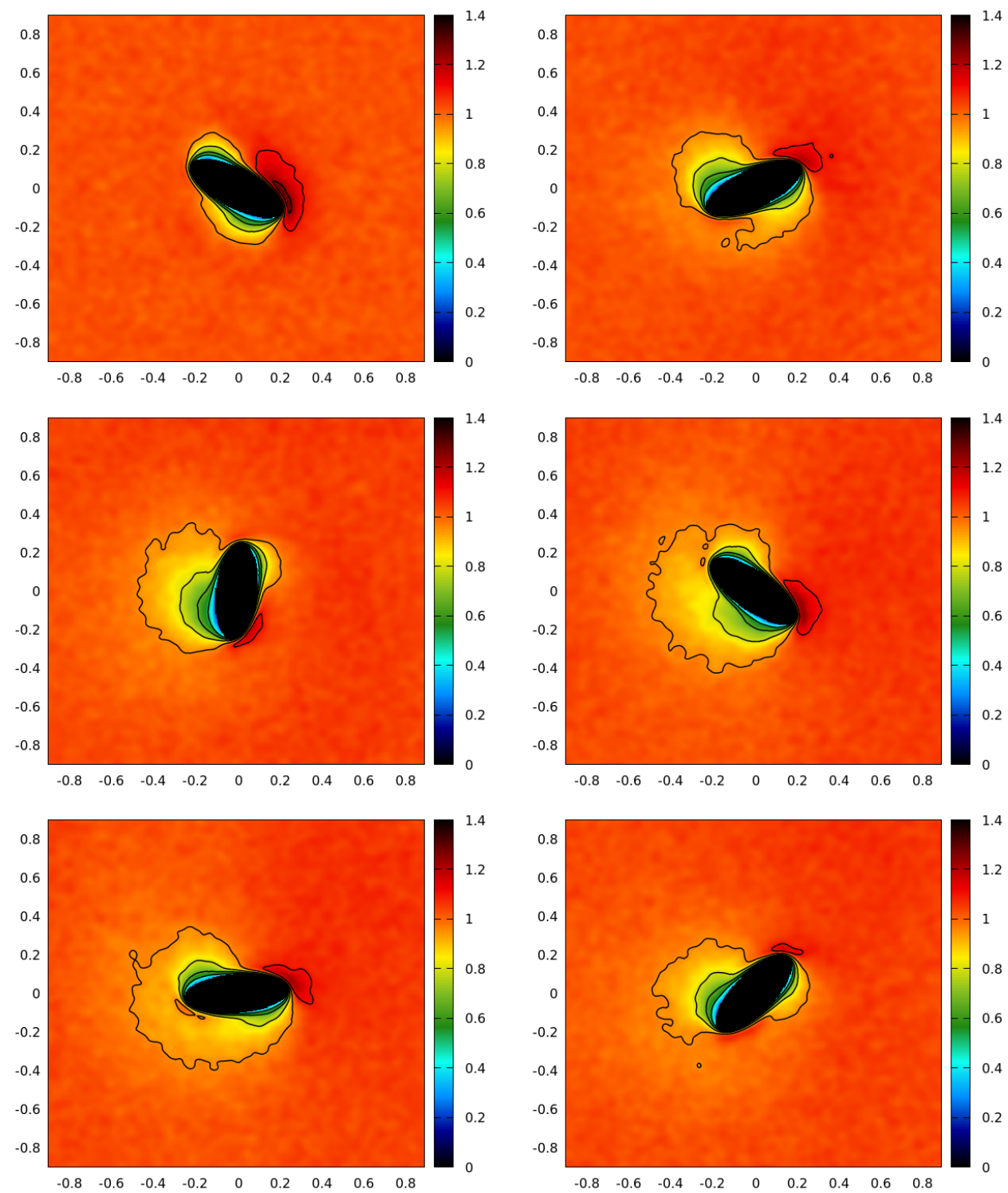

Figure 3: Time history of the gas density in Scenario 2, from left to right and from top to bottom, at times $8 \cdot 10^{-8} \mathrm{~s}, 2.4 \cdot 10^{-7} \mathrm{~s}, 4 \cdot 10^{-7} \mathrm{~s}, 5.6 \cdot 10^{-7} \mathrm{~s}, 7.2 \cdot 10^{-7}$ $\mathrm{s}, 8 \cdot 10^{-7} \mathrm{~s}$. The axis are scaled according to the length scale $L^{\circ}=10^{-4} \mathrm{~m}$.

rotational velocity, whereas in scenario 4 they have rotational velocities equal to $\Omega^{1}=2 \pi \cdot 10^{6} \mathrm{rad} / \mathrm{s}$ and $\Omega^{2}=-\pi \cdot 10^{6} \mathrm{rad} / \mathrm{s}$. Figures 5 and 6 show the evolution of the number density $\rho(t, x)=\int_{\mathbb{R}^{3}} f(t, x, v) \mathrm{d} v$ of scenario 3 and 4 respectively, and Figure 7 shows the time evolution of the kinetic temperature of the gas in scenario 4. 

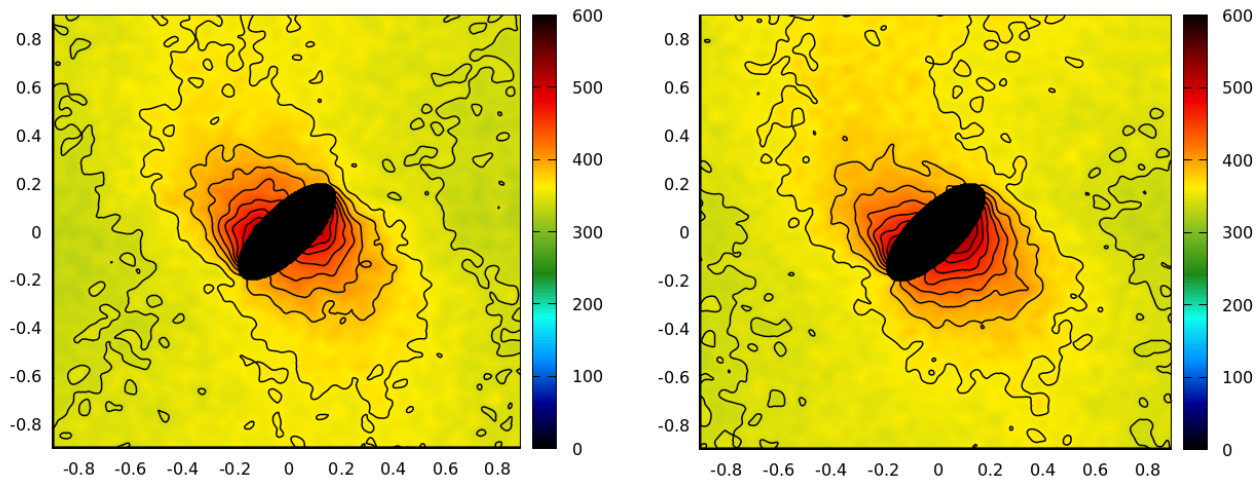

Figure 4: Temperature (in $\mathrm{K}$ ) at times $8 \cdot 10^{-7} \mathrm{~s}$ for scenario 1 (left) and for scenario 2 (right). The axis are scaled according to the length scale $L^{\circ}=10^{-4}$ $\mathrm{m}$.

\section{References}

[1] G. Allaire, X. Blanc, B. Despres, and F. Golse. Transport et diffusion. Editions de l'Ecole de polytechnique, 2018.

[2] K. Aoki and F. Golse. On the speed of approach to equilibrium for a collisionless gas. Kinet. Relat. Models, 4(1):87-107, 2011.

[3] G. Cavallaro and C. Marchioro. On the motion of an elastic body in a free gas. Reports on Mathematical Physics, 69(2):251-264, 2012.

[4] C. Cercignani. Rarefied gas dynamics: from basic concepts to actual calculations, volume 21. Cambridge University Press, 2000.

[5] F. Charles. Kinetic Modelling and Numerical Simulations using Particle Methods for the Transport of Dust in a Rarefied Gas. In Proceeding of the 26 th symposium Rarefied gas dynamics, volume 1084, pages 409-414. American Institute of Physics, 2009.

[6] F. Charles, C. Copol, S. Dellacherie, and J.-M. Mounsamy. Numerical simulation by a random particle method of deuterium-tritium reactions in a plasma. ESAIM PROCEEDINGS, 38:220-240, 2012.

[7] F. Charles and F. Salvarani. Mathematical and Numerical Study of a Dusty Knudsen Gas Mixture. Acta Applicandae Mathematicae, Jul 2019.

[8] F. De Vuyst and F. Salvarani. GPU-accelerated numerical simulations of the Knudsen gas on time-dependent domains. Comput. Phys. Commun., 184(3):532-536, 2013.

[9] G. Dechristé and L. Mieussens. Numerical simulation of micro flows with moving obstacles. In Journal of Physics: Conference Series, volume 362, page 012030. IOP Publishing, 2012. 

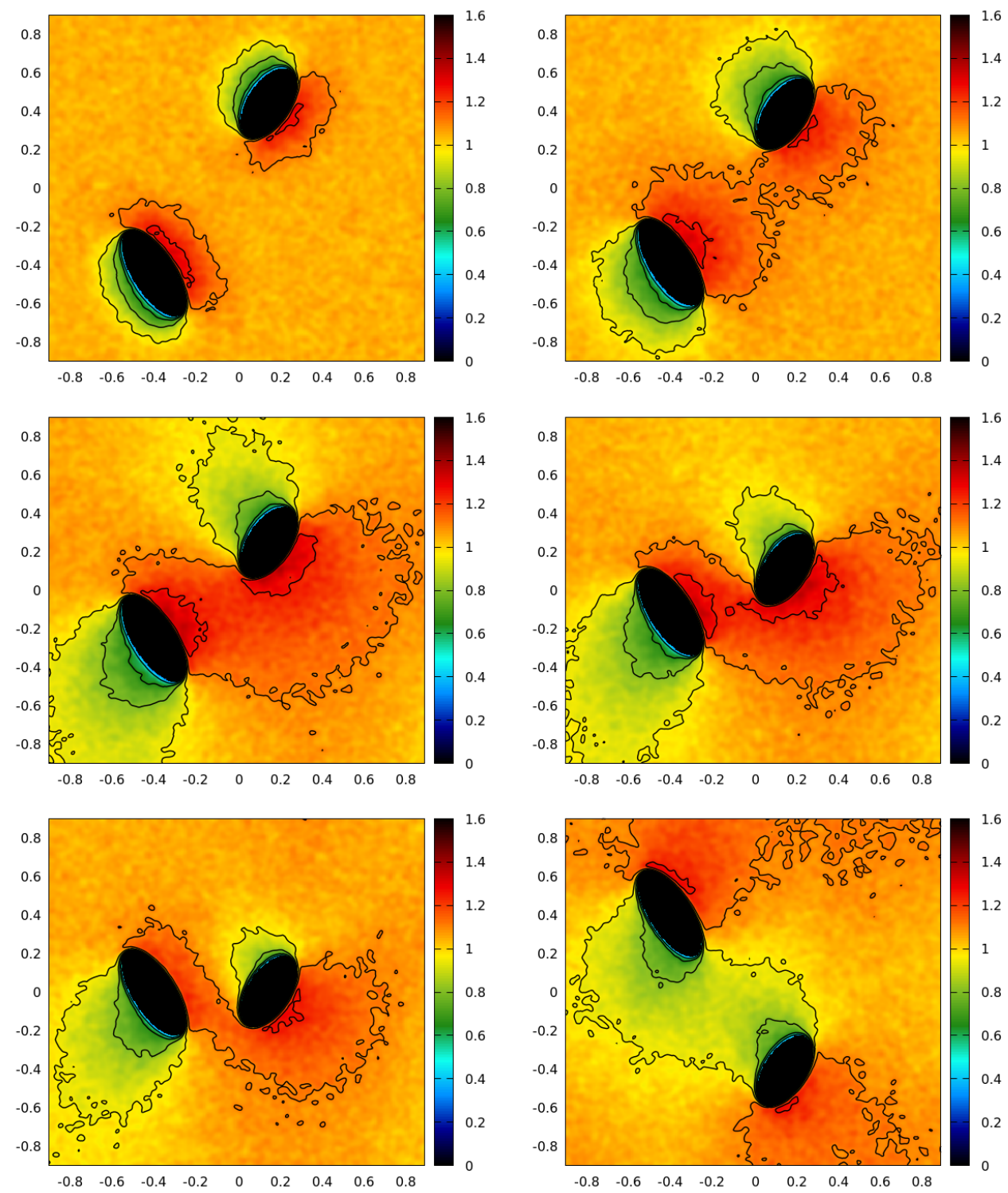

Figure 5: Time history of the gas density, from left to right and from top to bottom, in Scenario 3 at times $8 \cdot 10^{-8} \mathrm{~s}, 1.6 \cdot 10^{-7} \mathrm{~s}, 3.6 \cdot 10^{-7} \mathrm{~s}, 5.6 \cdot 10^{-7}$ $\mathrm{s}, 7.2 \cdot 10^{-7} \mathrm{~s}, 1.32 \cdot 10^{-6} \mathrm{~s}$. The axis are scaled according to the length scale $L^{\circ}=10^{-4} \mathrm{~m}$.

[10] N. M. Laurendeau. Statistical thermodynamics: fundamentals and applications. Cambridge University Press, 2005.

[11] S. Ostmo, A. Frezzotti, and T. Ytrehus. Kinetic theory study of steady evaporation from a spherical condensed phase containing inert solid particles. Physics of fluids, 9(1):211-225, 1997. 

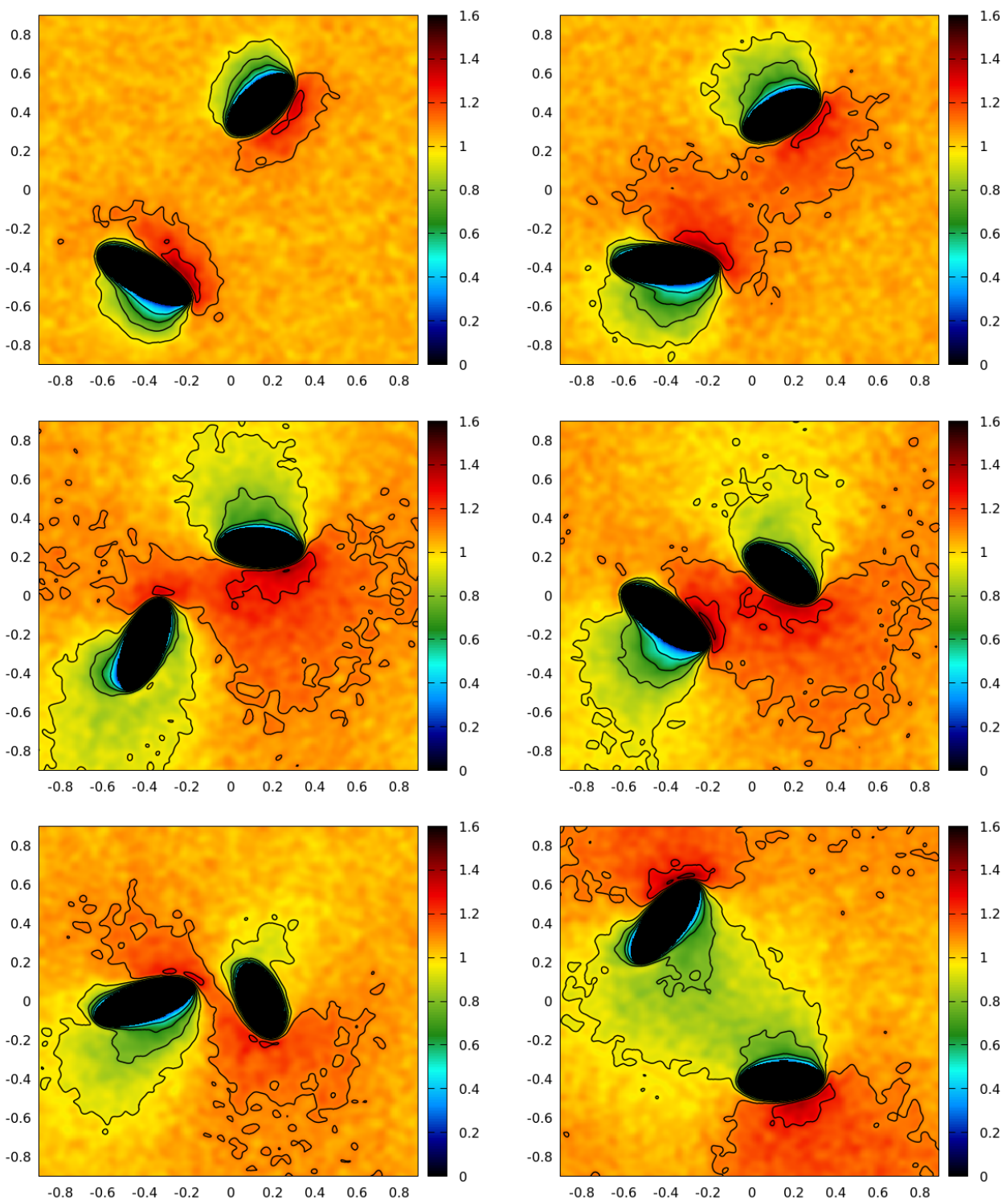

Figure 6: Time history of the gas density, from left to right and from top to bottom, in Scenario 4 at times $8 \cdot 10^{-8} \mathrm{~s}, 1.6 \cdot 10^{-7} \mathrm{~s}, 3.6 \cdot 10^{-7} \mathrm{~s}, 5.6 \cdot 10^{-7}$ $\mathrm{s}, 7.2 \cdot 10^{-7} \mathrm{~s}, 1.32 \cdot 10^{-6} \mathrm{~s}$. The axis are scaled according to the length scale $L^{\circ}=10^{-4} \mathrm{~m}$.

[12] G. Russo and F. Filbet. Semi-Lagrangian schemes applied to moving boundary problems for the bgk model of rarefied gas dynamics. 2009.

[13] S. Shrestha, S. Tiwari, A. Klar, and S. Hardt. Numerical simulation of moving rigid bodies in rarefied gases. J. Comp. Phys, 292:239-252, 2015.

[14] Y. Sone. Molecular gas dynamics. Modeling and Simulation in Science, 

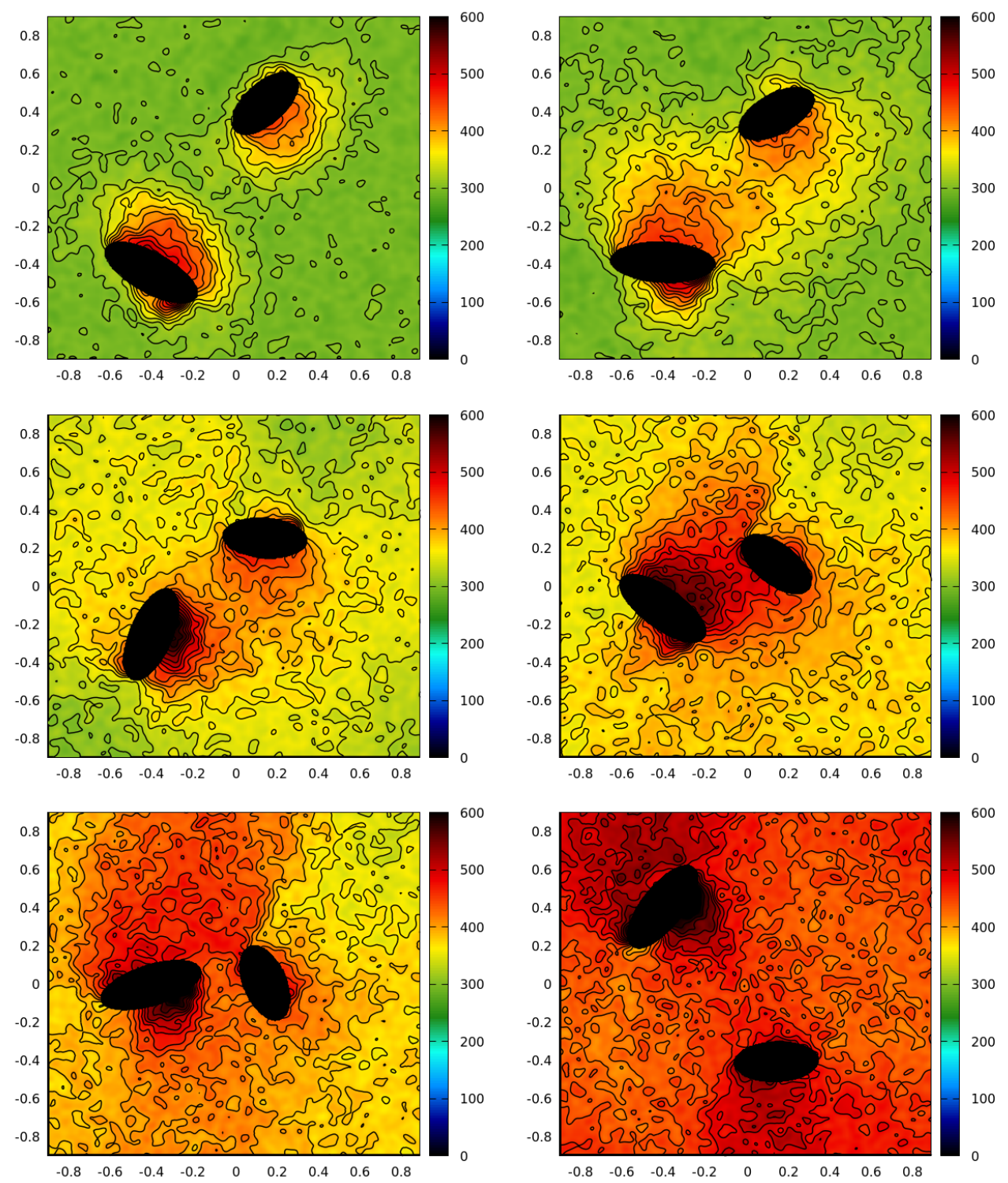

Figure 7: Time history of the temperature ( in $\mathrm{K}$ ) in Scenario 4, from left to right and from top to bottom, at times $8 \cdot 10^{-8} \mathrm{~s}, 1.6 \cdot 10^{-7} \mathrm{~s}, 3.6 \cdot 10^{-7} \mathrm{~s}$, $5.6 \cdot 10^{-7} \mathrm{~s}, 7.2 \cdot 10^{-7} \mathrm{~s}, 1.32 \cdot 10^{-6} \mathrm{~s}$. The axis are scaled according to the length scale $L^{\circ}=10^{-4} \mathrm{~m}$.

Engineering and Technology. Birkhäuser Boston, Inc., Boston, MA, 2007. Theory, techniques, and applications. 